\title{
Some Sufficient Conditions for Starlikeness and Convexity of Order $\alpha$
}

\author{
Yi-Ling Cang ${ }^{1}$ and Jin-Lin Liu ${ }^{2}$ \\ ${ }^{1}$ Department of Mathematics, Suqian College, Suqian 223800, China \\ ${ }^{2}$ Department of Mathematics, Yangzhou University, Yangzhou 225002, China \\ Correspondence should be addressed to Jin-Lin Liu; jlliu@yzu.edu.cn
}

Received 22 November 2012; Revised 12 February 2013; Accepted 16 February 2013

Academic Editor: Zhijun Liu

Copyright ( 2013 Y.-L. Cang and J.-L. Liu. This is an open access article distributed under the Creative Commons Attribution License, which permits unrestricted use, distribution, and reproduction in any medium, provided the original work is properly cited.

We derive certain sufficient conditions for starlikeness and convexity of order $\alpha$ of analytic functions in the unit disk. Applications are indicated for the subordination results to electromagnetic cloaking.

\section{Introduction}

Let $A(p)$ denote a class of functions $f(z)$ of the form

$$
f(z)=z^{p}+\sum_{n=1}^{\infty} a_{p+n} z^{p+n} \quad(p \in N=\{1,2,3, \ldots\}),
$$

which are analytic in the open unit $\operatorname{disk} U=\{z \in C:|z|<1\}$. When $p=1$, we write $A=A(1)$.

Let $f(z)$ and $g(z)$ be analytic in $U$. Then, the function $f(z)$ is said to be subordinate to $g(z)$, written as

$$
f(z) \prec g(z) \quad(z \in U),
$$

if there exists a Schwarz function $w(z)$ with $w(0)=0$ and $|w(z)|<1(z \in U)$ such that $f(z)=g(w(z))(z \in U)$. Furthermore, if the function $g(z)$ is univalent in $U$, then

$$
\begin{gathered}
f(z) \prec g(z) \quad(z \in U) \Longleftrightarrow f(0)=g(0), \\
f(U) \subset g(U) .
\end{gathered}
$$

A function $f(z)$ in $A(p)$ is said to be starlike of order $\alpha$ in $U$ if it satisfies

$$
\operatorname{Re}\left(\frac{z f^{\prime}(z)}{f(z)}\right)>\alpha \quad(z \in U)
$$

or equivalently

$$
\frac{z f^{\prime}(z)}{f(z)} \prec \frac{p+(p-2 \alpha) z}{1-z} \quad(z \in U)
$$

for some real $\alpha(0 \leq \alpha<p)$. We denote by $S_{p}^{*}(\alpha)$ the subclass of $A(p)$ consisting of all starlike functions of order $\alpha$ in $U$.

A function $f(z) \in A(p)$ is said to be convex of order $\alpha$ in $U$ if it satisfies

$$
\operatorname{Re}\left(1+\frac{z f^{\prime \prime}(z)}{f^{\prime}(z)}\right)>\alpha \quad(z \in U)
$$

or equivalently

$$
1+\frac{z f^{\prime \prime}(z)}{f^{\prime}(z)} \prec \frac{p+(p-2 \alpha) z}{1-z} \quad(z \in U)
$$

for some real $\alpha(0 \leq \alpha<p)$. We denote by $K_{p}(\alpha)$ the subclass of $A(p)$ consisting of all functions which are convex of order $\alpha$ in $U$. Also, we denote that $S_{p}^{*}(0)=S_{p}^{*}, S_{1}^{*}(0)=S^{*}$, and $K_{1}(0)=K$, respectively.

There are many results for conditions for $f(z) \in A$ to be in the classes $S^{*}$ and $K$ (e.g., see [1-11]). In the present paper, we aim at deriving some sufficient conditions for starlikeness and convexity of order $\alpha$ of functions in $A(p)$. In particular, we extend some related results obtained by several authors $[4-6,10]$. Possible applications of the subordination results to electromagnetic cloaking are also discussed in Section 3.

To derive our results, we need the following lemmas. 
Lemma 1 (see [3]). Let $p(z)$ be analytic and nonconstant in $U$ with $p(0)=1$. If $0<\left|z_{0}\right|<1$ and $\operatorname{Re} p\left(z_{0}\right)=$ $\min _{|z| \leq\left|z_{0}\right|} \operatorname{Re} p(z)$, then

$$
z_{0} p^{\prime}\left(z_{0}\right) \leq-\frac{\left|1-p\left(z_{0}\right)\right|^{2}}{2\left(1-\operatorname{Re} p\left(z_{0}\right)\right)} .
$$

Lemma 2 (see [4]). If $f(z) \in A$ satisfies

$$
\left|f^{\prime}(z)-1\right|<\frac{2}{\sqrt{5}} \quad(z \in U)
$$

then $f(z) \in S^{*}$.

Lemma 3 (see [4]). If $f(z) \in A$ satisfies

$$
\left|\arg f^{\prime}(z)\right|<\frac{\pi}{2} \delta \quad(z \in U)
$$

then $f(z) \in S^{*}$, where $\delta=0.6165 \ldots$ is the unique root of the equation

$$
2 \tan ^{-1}(1-\delta)+(1-2 \delta) \pi=0 .
$$

\section{Main Results}

Our first result is contained in the following.

Theorem 4. If $f(z) \in A(p)$ satisfies $f(z) f^{\prime}(z) \neq 0$ in $0<|z|<$ 1 and

$$
\left|\operatorname{Im}\left(\frac{z^{2} f^{\prime \prime}(z)}{f(z)}-\lambda \frac{z f^{\prime \prime}(z)}{f^{\prime}(z)}\right)\right|<\sqrt{\lambda(2 p+3 \lambda)} \quad(z \in U)
$$

for some real $\lambda(\lambda>0)$, then

$$
\frac{z f^{\prime}(z)}{f(z)} \prec p \frac{1+z}{1-z} \quad(z \in U)
$$

which is equivalent to $f(z) \in S_{p}^{*}$.

Proof. Let us define the analytic function $h(z)$ in $U$ by

$$
h(z)=\frac{z f^{\prime}(z)}{p f(z)} .
$$

Then, $h(0)=1, h(z) \neq 0$, and

$$
\begin{aligned}
\frac{z^{2} f^{\prime \prime}(z)}{f(z)}-\lambda \frac{z f^{\prime \prime}(z)}{f^{\prime}(z)} & =\frac{z f^{\prime \prime}(z)}{f^{\prime}(z)}\left(\frac{z f^{\prime}(z)}{f(z)}-\lambda\right) \\
& =(p h(z)-\lambda)\left(\frac{z h^{\prime}(z)}{h(z)}+h(z)-1\right) .
\end{aligned}
$$
that

Suppose that there exists a point $z_{0}\left(0<\left|z_{0}\right|<1\right)$ such

$$
\operatorname{Re} h(z)>0 \quad\left(|z|<\left|z_{0}\right|\right), \quad h\left(z_{0}\right)=i \beta
$$

where $\beta$ is real and $\beta \neq 0$. Then, applying Lemma 1 , we get

$$
z_{0} h^{\prime}\left(z_{0}\right) \leq-\frac{1+\beta^{2}}{2} .
$$

Thus, it follows from (15), (16), and (17) that

$$
\begin{aligned}
I_{0} & =\operatorname{Im}\left(\frac{z_{0}^{2} f^{\prime \prime}\left(z_{0}\right)}{f\left(z_{0}\right)}-\lambda \frac{z_{0} f^{\prime \prime}\left(z_{0}\right)}{f^{\prime}\left(z_{0}\right)}\right) \\
& =-\beta(p+\lambda)+\frac{\lambda}{\beta} z_{0} h^{\prime}\left(z_{0}\right) .
\end{aligned}
$$

In view of $\lambda>0$, from (17) and (18), we obtain

$$
\begin{gathered}
I_{0} \geq-\frac{\lambda+(2 p+3 \lambda) \beta^{2}}{2 \beta} \geq \sqrt{\lambda(2 p+3 \lambda)} \quad(\beta<0), \\
I_{0} \leq-\frac{\lambda+(2 p+3 \lambda) \beta^{2}}{2 \beta} \leq-\sqrt{\lambda(2 p+3 \lambda)} \quad(\beta>0) .
\end{gathered}
$$

But both (19) and (20) contradict assumption (12). Therefore, we must have $\operatorname{Re} h(z)>0(z \in U)$; that is,

$$
\frac{z f^{\prime}(z)}{f(z)} \prec p \frac{1+z}{1-z} \quad(z \in U) .
$$

The proof of the theorem is complete.

By taking $p=\lambda=1$ in Theorem 4, we have the following result which is due to Lin and Owa [2].

Corollary 5. If $f(z) \in A$ satisfies $f(z) f^{\prime}(z) \neq 0$ in $0<|z|<1$ and

$$
\left|\operatorname{Im}\left\{\frac{z^{2} f^{\prime \prime}(z)}{f^{\prime}(z)}-\frac{z f^{\prime \prime}(z)}{f^{\prime}(z)}\right\}\right|<\sqrt{5} \quad(z \in U),
$$

then $f(z) \in S^{*}$.

Next, we derive the following.

Theorem 6. If $f(z) \in A(p)$ satisfies

$$
\begin{gathered}
\left|\left(\frac{f(z)}{z^{p}}\right)^{1 /(p-\alpha)}\left(\frac{z f^{\prime}(z)}{f(z)}-\alpha\right)-p+\alpha\right| \\
<\frac{2}{\sqrt{5}}(p-\alpha) \quad(z \in U)
\end{gathered}
$$

for some real $\alpha(0 \leq \alpha<p)$, then

$$
\frac{z f^{\prime}(z)}{f(z)} \prec \frac{p+(p-2 \alpha) z}{1-z} \quad(z \in U),
$$

or equivalently $f(z) \in S_{p}^{*}(\alpha)$.

Proof. For $f(z) \in A(p)$, we define the function $p(z)$ by

$$
p(z)=\left(\frac{f(z)}{z^{\alpha}}\right)^{1 /(p-\alpha)}=z+\cdots .
$$


Then, $p(z)$ is analytic in $U$, and

$$
\begin{aligned}
\left|p^{\prime}(z)-1\right| & =\frac{1}{p-\alpha}\left|\left(\frac{f(z)}{z^{p}}\right)^{1 /(p-\alpha)}\left(\frac{z f^{\prime}(z)}{f(z)}-\alpha\right)-p+\alpha\right| \\
& <\frac{2}{\sqrt{5}} \quad(z \in U)
\end{aligned}
$$

according to the condition of the theorem. By using Lemma 2, we have that $p(z) \in S^{*}$.

Note that

$$
\frac{z p^{\prime}(z)}{p(z)}=\frac{1}{p-\alpha}\left(\frac{z f^{\prime}(z)}{f(z)}-\alpha\right) .
$$

This shows that

$$
\frac{z f^{\prime}(z)}{f(z)} \prec \frac{p+(p-2 \alpha) z}{1-z} \quad(z \in U) ;
$$

that is, $f(z) \in S_{p}^{*}(\alpha)$. The proof of the theorem is complete.

Theorem 7. If $f(z) \in A(p)$ satisfies

$$
\begin{aligned}
\mid \arg & \left(\frac{f(z)}{z^{p}}\right)+(p-\alpha) \arg \left(\frac{z f^{\prime}(z)}{f(z)}-\alpha\right) \mid \\
& <\frac{\pi}{2}(p-\alpha) \delta \quad(z \in U)
\end{aligned}
$$

for some real $\alpha(0 \leq \alpha<p)$, then

$$
\frac{z f^{\prime}(z)}{f(z)} \prec \frac{p+(p-2 \alpha) z}{1-z} \quad(z \in U),
$$

or equivalently $f(z) \in S_{p}^{*}(\alpha)$, where $\delta=0.6165 \ldots$ is the unique root of the equation

$$
2 \tan ^{-1}(1-\delta)+(1-2 \delta) \pi=0 .
$$

Proof. Let us define the function $p(z)$ as in (25). Then, we have

$$
\begin{aligned}
\arg p^{\prime}(z) & =\arg \left(\frac{1}{p-\alpha}\left(\frac{f(z)}{z^{p}}\right)^{1 /(p-\alpha)}\left(\frac{z f^{\prime}(z)}{f(z)}-\alpha\right)\right) \\
& =\frac{1}{p-\alpha} \arg \left(\frac{f(z)}{z^{p}}\right)+\arg \left(\frac{z f^{\prime}(z)}{f(z)}-\alpha\right) .
\end{aligned}
$$

In view of Lemma 3, we see that if

$$
\begin{aligned}
& \frac{1}{p-\alpha}\left|\arg \left(\frac{f(z)}{z^{p}}\right)+(p-\alpha) \arg \left(\frac{z f^{\prime}(z)}{f(z)}-\alpha\right)\right| \\
& <\frac{\pi}{2} \delta \quad(z \in U),
\end{aligned}
$$

then $p(z) \in S^{*}$. This shows that $f(z) \in S_{p}^{*}(\alpha)$.

Finally, we discuss the following theorem.
Theorem 8. If $f(z) \in A(p)$ satisfies

$$
\begin{aligned}
& \left|\arg \left(\frac{f^{\prime}(z)}{z^{p-1}}\right)+(p-\alpha) \arg \left(1+\frac{z f^{\prime \prime}(z)}{f^{\prime}(z)}-\alpha\right)\right| \\
& \quad<\frac{\pi}{2}(p-\alpha) \delta \quad(z \in U)
\end{aligned}
$$

for some real $\alpha(0 \leq \alpha<p)$, then

$$
1+\frac{z f^{\prime \prime}(z)}{f^{\prime}(z)} \prec \frac{p+(p-2 \alpha) z}{1-z} \quad(z \in U),
$$

or equivalently $f(z) \in K_{p}(\alpha)$, where $\delta=0.6165 \ldots$ is the unique root of the equation

$$
2 \tan ^{-1}(1-\delta)+(1-2 \delta) \pi=0 .
$$

Proof. For $f(z) \in A(p)$, we define the function $p(z)$ by

$$
p(z)=\int_{0}^{z}\left(\frac{f^{\prime}(t)}{p t^{p-1}}\right)^{1 /(p-\alpha)} d t=z+\cdots .
$$

Then, $p(z)$ is analytic in $U$. Further, letting $g(z)=z p^{\prime}(z)$, we obtain that

$$
g^{\prime}(z)=\frac{1}{p-\alpha}\left(\frac{f^{\prime}(z)}{p z^{p-1}}\right)^{1 /(p-\alpha)}\left(1+\frac{z f^{\prime \prime}(z)}{f^{\prime}(z)}-\alpha\right) .
$$

Thus, applying Lemma 3, we have that

$$
\begin{aligned}
& \mid \arg \left(g^{\prime}(z)\right) \mid \\
&=\left|\frac{1}{p-\alpha} \arg \left(\frac{f^{\prime}(z)}{z^{p-1}}\right)+\arg \left(1+\frac{z f^{\prime \prime}(z)}{f^{\prime}(z)}-\alpha\right)\right| \\
& \quad<\frac{\pi}{2} \delta
\end{aligned}
$$

for $z \in U$, which shows that $g(z) \in S^{*}$. This gives us that $p(z) \in K$; that is, $f(z) \in K_{p}(\alpha)$.

\section{Applications}

To make objects invisible to human eyes has been long for a subject of science fiction. But just in 2006, this imagination has been materialized in the range of microwave radiation. This is attributed to pioneering papers published in Science by Leonhardt [12] and Pendry et al. [13] in 2006, in which they proposed an ingenious idea to control electromagnetic waves by specially designed materials. They suggest that a cloak, made of metamaterial (in which the refractive index spatially varies), can be designed so that an incident electromagnetic wave can be guided through the cloak giving an impression of free space when viewed from outside. This ensures that the cloak neither reflects nor scatters waves nor casts a shadow in the transmitted field. The cloak remains undetected by a viewing device. At the same time, the cloak reduces scattering of radiation from the object where the imperfections are 
exponentially small. Hence, the object becomes invisible to the detector.

Reports are available in the published literature (e.g., see $[12,13])$ that electromagnetic cloaking, which seemed impossible earlier, is technologically realizable when the cloak and the cloaked object have a circular symmetry in at least one plane, namely, spheres and cylinders. The cross-section is a laminar or two-dimensional cloaking. For some important research contributions on this subject, see, for example, [1417].

Mathematically, the two-dimensional cloak and the cloaked object are simply connected regions in the complex plane, the latter being a subset of the former. By the Riemann mapping theorem, both regions are equivalent to conformal maps on the unit disk $U$. If we denote the cloaked object by the function $g(z)$ and the cloak by the function $q(z)$, then it is required that

$$
g(z) \prec q(z) \quad(z \in U) .
$$

Very recently, Mishra et al. [18] have given some applications of subordination relationship (40) to electromagnetic cloaking. In the present paper, we found several sufficient conditions under which relationship of the form (40) holds for functions which are more general than circular maps. For example, in Theorem 4, we have taken the cloak function

$$
q(z)=p \frac{1+z}{1-z}
$$

to be an analytic univalent convex map. If a function $f(z)$ satisfies condition (12), then the subordination relationship (40) holds true.

\section{Acknowledgment}

The authors would like to express sincere thanks to the referees for careful reading and suggestions which helped them to improve the paper.

\section{References}

[1] J.-L. Li and S. Owa, "Sufficient conditions for starlikeness," Indian Journal of Pure and Applied Mathematics, vol. 33, no. 3, pp. 313-318, 2002.

[2] L. J. Lin and S. Owa, "Properties of the Salagean operator," Georgian Mathematical Journal, vol. 5, no. 4, pp. 361-366, 1998.

[3] S. S. Miller and P. T. Mocanu, "Second-order differential inequalities in the complex plane," Journal of Mathematical Analysis and Applications, vol. 65, no. 2, pp. 289-305, 1978.

[4] P. T. Mocanu, "Some starlikeness conditions for analytic functions," Revue Roumaine de Mathématiques Pures et Appliquées, vol. 33, no. 1-2, pp. 117-124, 1988.

[5] P. T. Mocanu, "Some simple criteria for starlikeness and convexity," Libertas Mathematica, vol. 13, pp. 27-40, 1993.

[6] M. Nunokawa, S. Owa, Y. Polatoglu, M. Caglar, and E. Y. Duman, "Some sufficient conditions for starlikeness and convexity," Turkish Journal of Mathematics, vol. 34, no. 3, pp. 333-337, 2010.
[7] C. Ramesha, S. Kumar, and K. S. Padmanabhan, "A sufficient condition for starlikeness," Chinese Journal of Mathematics, vol. 23, no. 2, pp. 167-171, 1995.

[8] J. Sokó1, "On an application of certain sufficient condition for starlikeness," Journal of Mathematics and Applications, vol. 30, pp. 131-135, 2008.

[9] J. Sokól, “On a condition for $\alpha$-starlikeness," Journal of Mathematical Analysis and Applications, vol. 352, no. 2, pp. 696-701, 2009.

[10] N. Uyanık, M. Aydogan, and S. Owa, "Extensions of sufficient conditions for starlikeness and convexity of order $\alpha$," Applied Mathematics Letters, vol. 24, no. 8, pp. 1393-1399, 2011.

[11] N. Xu and D. Yang, "Some criteria for starlikeness and strongly starlikeness," Bulletin of the Korean Mathematical Society, vol. 42, no. 3, pp. 579-590, 2005.

[12] U. Leonhardt, “Optical conformal mapping," Science, vol. 312, no. 5781, pp. 1777-1780, 2006.

[13] J. B. Pendry, D. Schurig, and D. R. Smith, "Controlling electromagnetic fields," Science, vol. 312, no. 5781, pp. 1780-1782, 2006.

[14] N. Kundtz and D. R. Smith, "Extreme-angle broadband metamaterial lens," Nature Materials, vol. 9, pp. 129-132, 2010.

[15] R. Liu, C. Ji, J. J. Mock, J. Y. Chin, T. J. Cui, and D. R. Smith, "Broadband ground-plane cloak," Science, vol. 323, no. 5912, pp. 366-369, 2009.

[16] G. A. Rao and S. P. Mahulikar, "Integrated review of stealth technology and its role in airpower," Aeronautical Journal, vol. 106, pp. 629-641, 2002.

[17] D. Schurig, J. J. Mock, B. J. Justice et al., "Metamaterial electromagnetic cloak at microwave frequencies," Science, vol. 314, no. 5801, pp. 977-980, 2006.

[18] A. K. Mishra, T. Panigrahi, and R. K. Mishra, "Subordination and inclusion theorems for subclasses of meromorphic functions with applications to electromagnetic cloaking," Mathematical and Computer Modelling, vol. 57, pp. 945-962, 2013. 


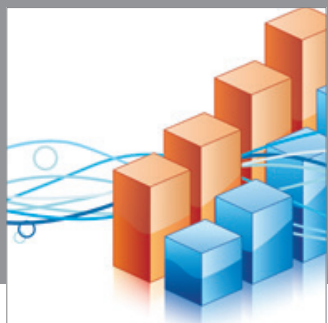

Advances in

Operations Research

mansans

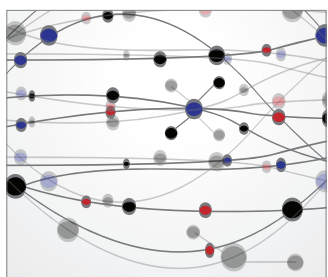

The Scientific World Journal
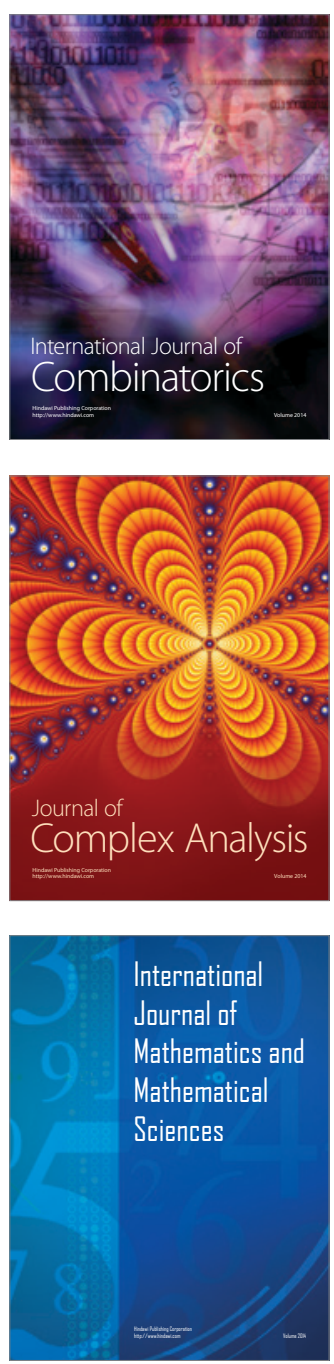
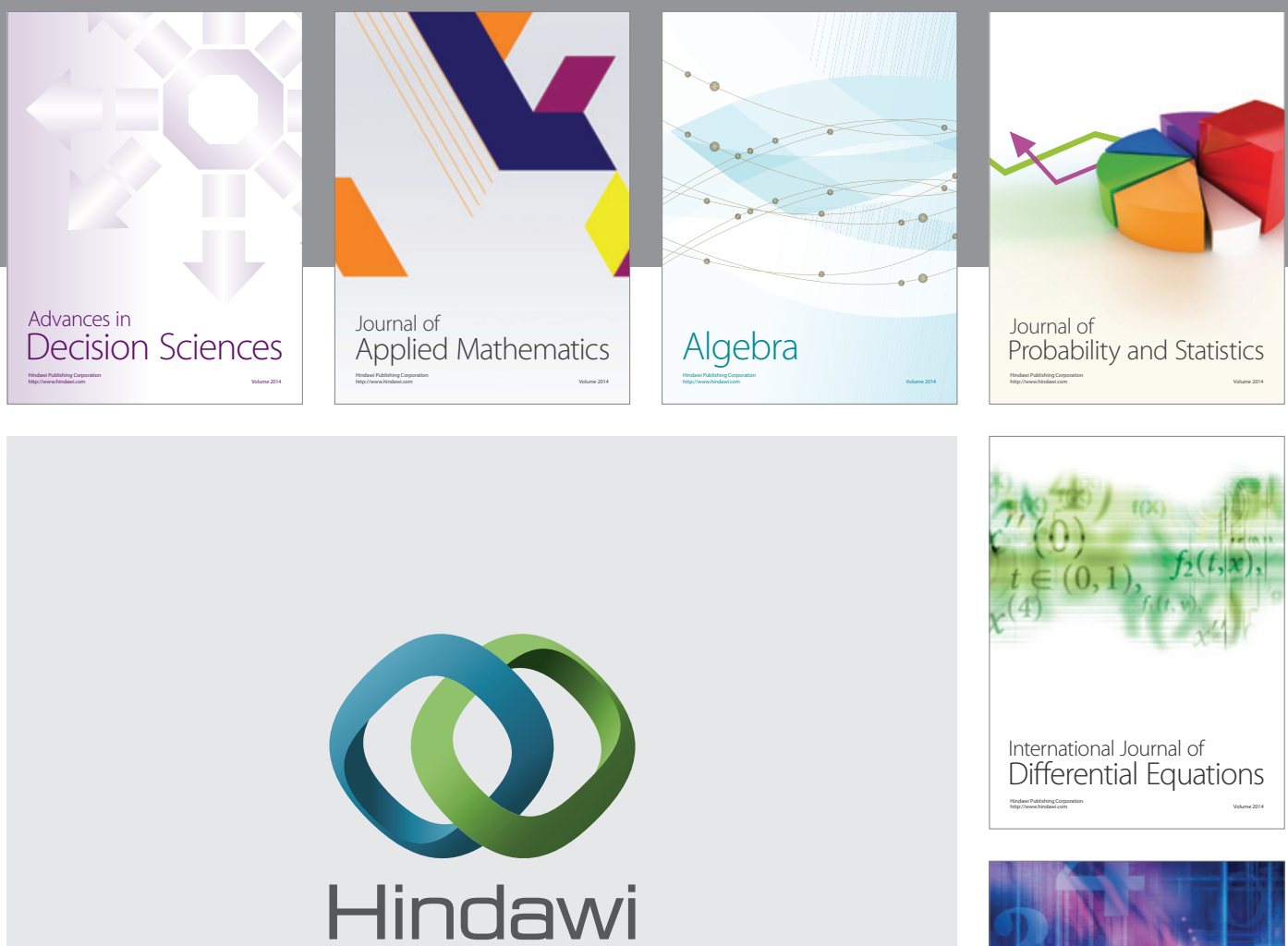

Submit your manuscripts at http://www.hindawi.com
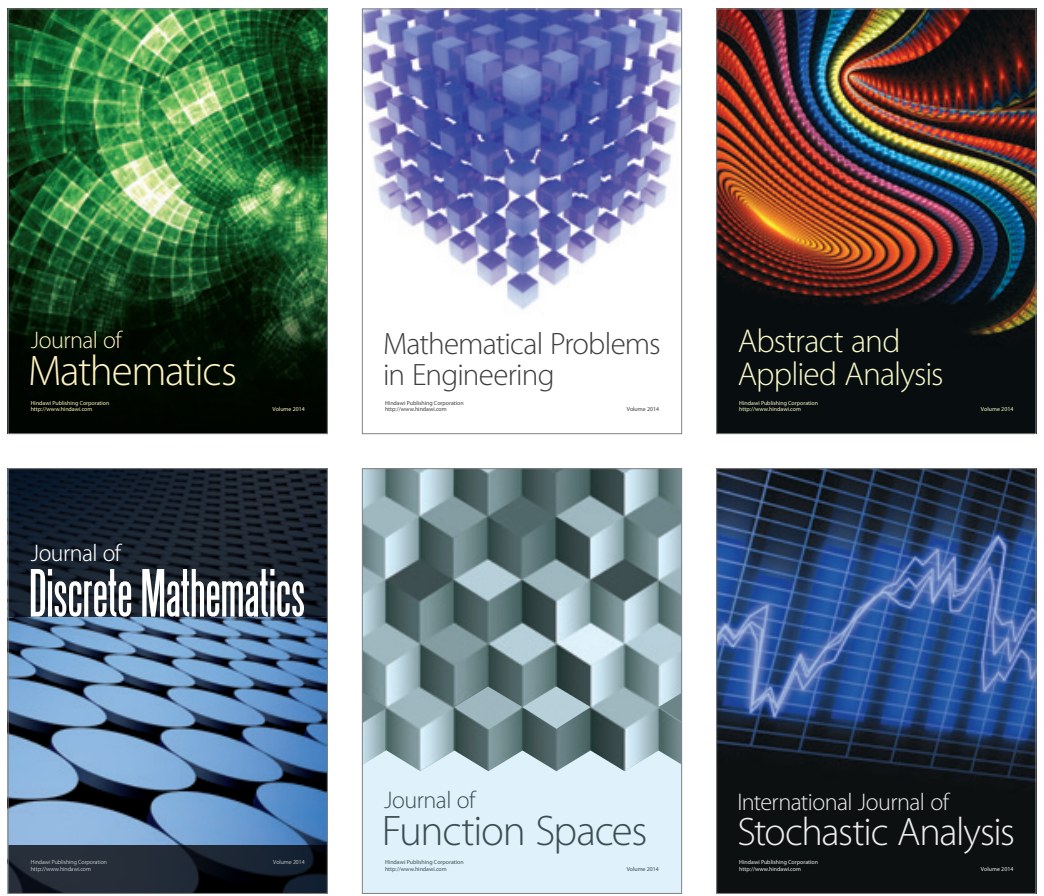

Journal of

Function Spaces

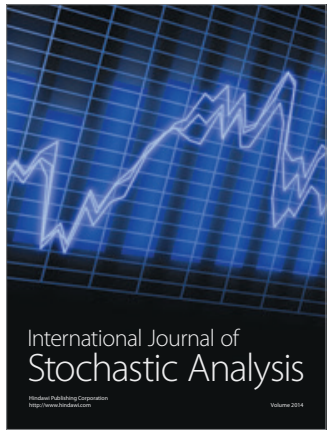

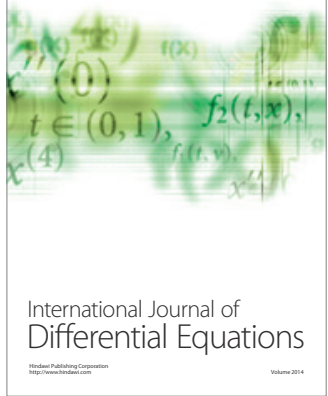
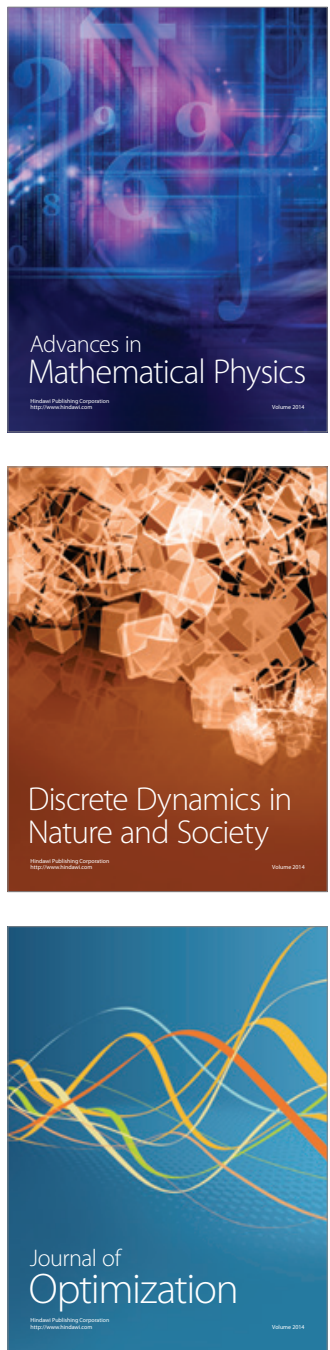